\title{
Alterações degenerativas em pacientes com disfunção crâniomandibular
}

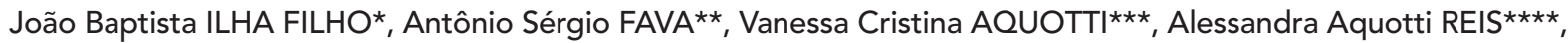

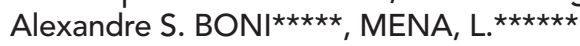

\begin{abstract}
Resumo
Os processos degenerativos da ATM foram estudados em 17 pacientes, 34 ATMs do Serviço de Cirurgia Bucomaxilofacial do Hospital Jaraguá-São Paulo/SP, todos com diagnóstico de disfunção crâniomandibular. Foi utilizado o exame radiográfico do tipo transcraniano para verificação das degenerações articulares. As ATMs de cada paciente foram examinadas por três diferentes profissionais com auxílio do exame radiográfico. O exame clínico procurou observar dados como raça, gênero, a queixa principal de cada paciente, presença de dor pré-auricular, dor muscular, estalo nas articulações, e limitação da abertura da boca. A dor pré-auricular, e os estalos foram as principais queixas dos pacientes. As alterações degenerativas ocorreram mais nas mulheres com faixa etária entre 20 a 29 anos. Ocorreram na seguinte ordem decrescente: facetamento, eburnação e osteófito. As alterações foram mais marcantes no côndilo do que na eminência articular, já os facetamentos estiveram relacionados com a queixa principal de dor pré-auricular. Foi observado estatisticamente uma correlação de $70 \%$ entre o facetamento e a ebunação.
\end{abstract}

Palavras-chave: Articulação temporomandibular. Disfunções. Radiologia.

\section{INTRODUÇÃO}

Os processos degenerativos da articulação (ATM) estão relacionados com seu mau funcionamento, e os exames radiológicos são freqüentemente incluídos no diagnóstico e estadiamento das disfunções crâniomandibulares (DCM). As degenerações das ATMs são muito freqüentes em pacientes idosos, verificadas através de material de necropsial.

Os sons da ATM como o estalido são indicativos da falta de sincronismo entre o côndilo da mandíbula e disco articular, podendo acarretar alterações degenerativas ${ }^{3}$. As crepitações são indicativos de atrito entre as fibro-cartilagens, ocorrendo

\footnotetext{
* Cirurgião bucomaxilofacial, Mestrando em Ciências da Saúde do Hospital Heliópolis SP, Prof. da disciplina da graduação e especialização de Cirurgia e Traumatologia da Faculdade Ingá, Maringá-PR.

** Médico Cirurgião Cabeça e Pescoço, Docente do curso de Mestrado em Ciências da Saúde do Hospital Heliópolis-SP.

*** Cirurgiã bucomaxilofacial, Mestranda em Ciências da Saúde do Hospital Heliópolis SP.

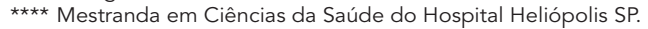

***** Cirurgião bucomaxilofacial, Mestrando em Ciências da Saúde do Hospital Heliópolis SP.

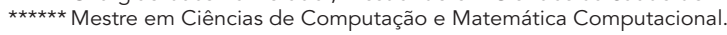


devido às superfícies estarem irregulares, e têm sido consideradas como representantes da doença avançada. A crepitação pode indicar perfuração do disco articular ou inserção, especialmente se há imagem degenerativa ${ }^{12}$.

As principais alterações degenerativas das ATMs compreendem o facetamento, a erosão, o osteófito, a esclerose, a concavidade e o pseudocisto $^{5,17}$.

Bean et $\mathrm{al} .{ }^{4}$ observaram várias articulações com erosões ao exame radiográfico, exibindo extensa destruição do osso compacto subarticular de forma achatada definida como facetamento, apesar de não ter sido observado macroscopicamente alterações nas superfícies dos tecidos moles das ATMs.

Existe controvérsia quanto ao significado das alterações degenerativas, pois pacientes assintomáticos podem ser acometidos por essas alterações, da mesma forma que os pacientes com DCMs ${ }^{8}$.

O exame radiográfico é fundamental no exame clínico da ATM, estando a cada dia mais sofisticados, como a artrografia, tomografia computadorizada, tomografia helicoidal, ressonância magnética e a axiossonomiografia computadorizada ${ }^{12}$. Entretanto os métodos radiográficos convencionais também podem ser úteis na avaliação das ATMs. As radiografias convencionais mais utilizadas pelos clínicos são as laterais transcranianas e laterais transfaciais preconizadas e descritas ${ }^{15}$. Estes métodos são menos onerosos e, também, podem ser utilizados na maioria dos ambulatórios de cirurgia bucomaxilofacial com a utilização de contrastes para a verificação do disco articular.

A utilização da radiografia transcranial convencional tem limites devido à sua realização em apenas um plano, além da ocorrência de distorções radiográficas. É um exame radiológico valioso por ser acessível em muitos serviços públicos, e apesar de apresentar algumas deficiências permite uma boa visualização do côndilo mandibular e do tubérculo articular, mesmo quando comparada à tomografia linear ${ }^{2}$. O exame radiográfico transcra- niano, quando comparado com os demais exames da ATM, pode ser considerado de grande valor no exame de alterações da excursão condilar e degenerações articulares.

Moraes et al. ${ }^{16}$ compararam diferentes tipos de exames radiográficos da ATM e concluiram que o exame transcraniano só foi superado pela tomografia linear para visualização da degeneração articular. É o melhor exame radiográfico para visualização da hipermobilidade articular ou hiperexcursão articular acentuada, superando inclusive a ressonância nuclear magnética.

A tomografia computadorizada não permite imagem sagital da ATM, devido à posição da cabeça do paciente no aparelho. Sendo assim a tomografia linear apresenta vantagem na visualização da superfície óssea, seguido do exame transcraniano.

A proposta desse estudo é investigar os aspectos macroscópicos degenerativos de 34 ATMs, nos achados radiográficos transcranianos.

\section{CASUÍSTICA E MÉTODO Casuística}

Foram avaliados 17 pacientes (34 ATMs), no serviço de Cirurgia Bucomaxilofacial do Hospital Jaraguá, São Paulo-SP, todos com diagnóstico prévio de DCM.

O estudo foi aprovado pelo comitê de ética na pesquisa do Hospital Heliópolis-SP sob o número 241 e realizado de acordo com a resolução 196/96 de 10 outubro 1996. Todos os pacientes assinaram o consetimento livre e esclarecido para realização deste trabalho.

\section{MÉTODO Investigação clínica}

$\mathrm{Na}$ investigação clínica foram avaliadas as características de cada paciente quanto à idade, raça, gênero, profissão e anotada a data do atendimento e o endereço para contato. Com auxílio de uma ficha clínica foram avaliadas a queixa principal, a presença de dor pré-auricular, de dor muscular, de má oclusão, dos hábitos parafun- 
cionais e dos estalos e crepitações das ATMs. A limitação da abertura bucal (trismo), a presença de luxação recidivante, de zumbido no ouvido, da otalgia e tensão emocional também foram avaliadas e registradas na ficha de atendimento. Foram excluídos da pesquisa pacientes usuários de prótese total dupla, pacientes que tinham sofrido algum tipo de trauma na região da articulação e incluídos os pacientes com próteses parciais removíveis, com ausências dentárias de um ou mais dentes e de ambos os gêneros.

O exame clínico foi realizado através da palpação muscular bilateral extra-oral e intra-oral. Essa avaliação muscular visa observar se existe dor muscular associada às alterações degenerativas da ATM.

$\mathrm{Na}$ palpação extra-oral foram avaliados os seguintes músculos: masseter superficial, masseter profundo, temporal anterior, temporal posterior, região frontal, região de vértex, cervicais posteriores, digástrico e esternocleidomastoídeo.

Quanto à palpação da musculatura intra-oral foi verificado: a inserção do temporal, pterigoi-

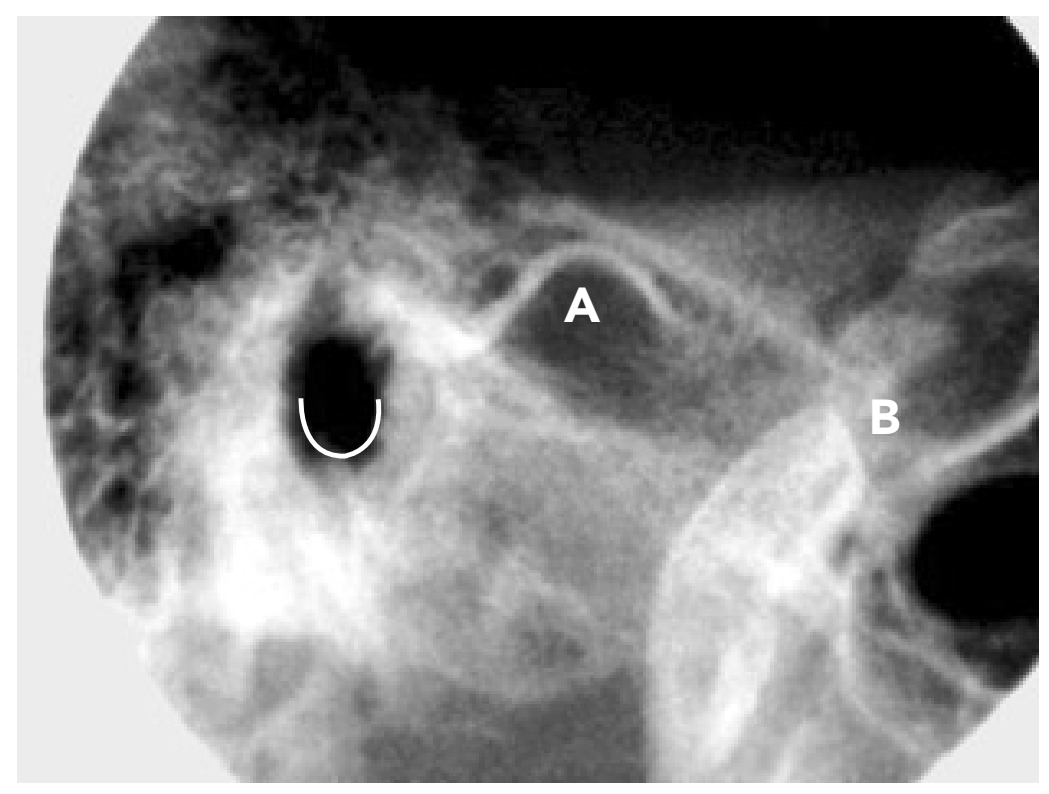

FIGURA 1 - A) Eburnação na cavidade glenóide; B) Facetamento no côndilo. deo lateral e pterigoideo medial.

Foi realizada a palpação da musculatura facial intra-oral e extra-oral com o dedo indicador com leve pressão, de acordo com os trabalhos de Helkimo $^{10}$.

A palpação da ATM para verificação de dor lateral e posterior à cápsula articular foi realizada com o dedo indicador de uma das mãos com suave pressão ${ }^{10,19}$.

A auscultação das articulações foi realizada cuidadosamente com auxílio de um estetoscópio da marca Littman classic de fabricação norte americana.

\section{Investigação radiológica}

Foram realizadas tomadas radiográficas transcranianas de todos os pacientes com diagnóstico de DCM em posição de repouso com a boca fechada e com a boca em máxima abertura ${ }^{22}$.

O exame radiográfico verificou as degenerações das ATMs como: facetamento, eburnação, osteófito, pseudocisto e erosões com auxílio de mais três profissionais, que utilizaram os mesmos critérios durante a avaliação. Facetamento é a perda da convexidade ou aplainamento dos côndilos ou eminências articulares (Fig. 1). A eburnação ocorre quando a fibrocartilagem do côndilo sofre um atrito contra a fibrocartilagem da cavidade glenóide possuindo uma radiopacidade aumentada (Fig. 2). Pseudocisto é uma área radiolúcida abaixo da superfície articular. Osteófito é uma elevação na superfície articular (Fig. 3). E a erosão é uma região localizada de rarefação óssea ou reabsorção óssea.

Os resultados das degenerações foram avaliados em cada exame radiográfico e os resul- 


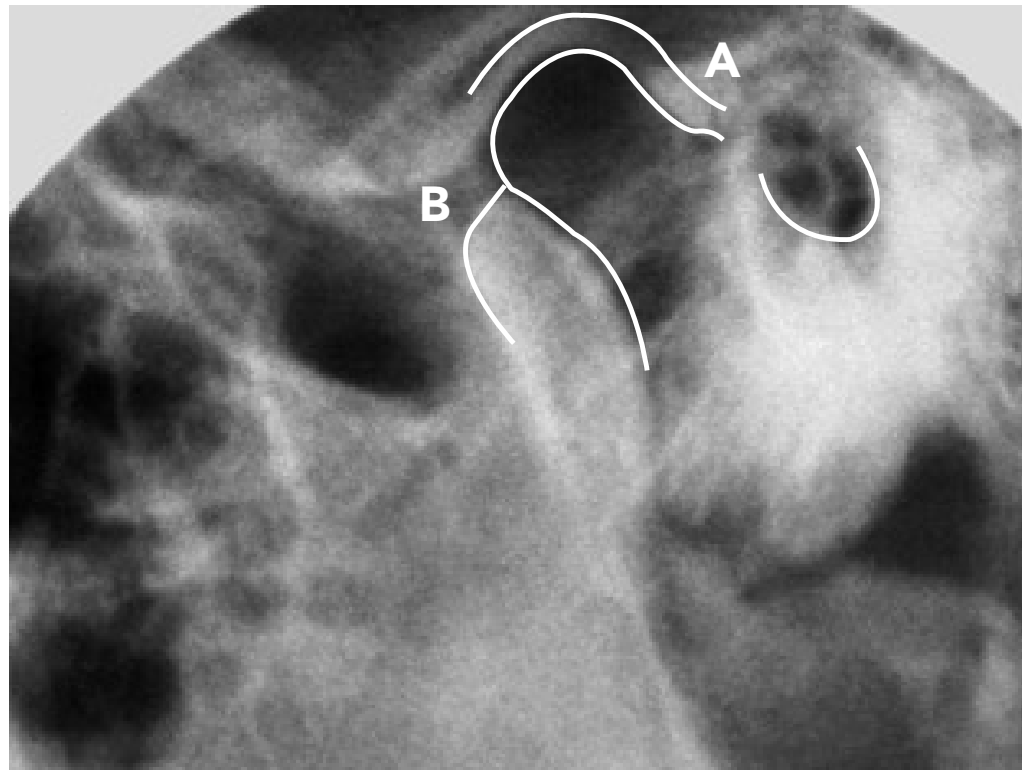

FIGURA 2 - A) Eburnação na eminência e no côndilo; B) Côndilo com facetamento.

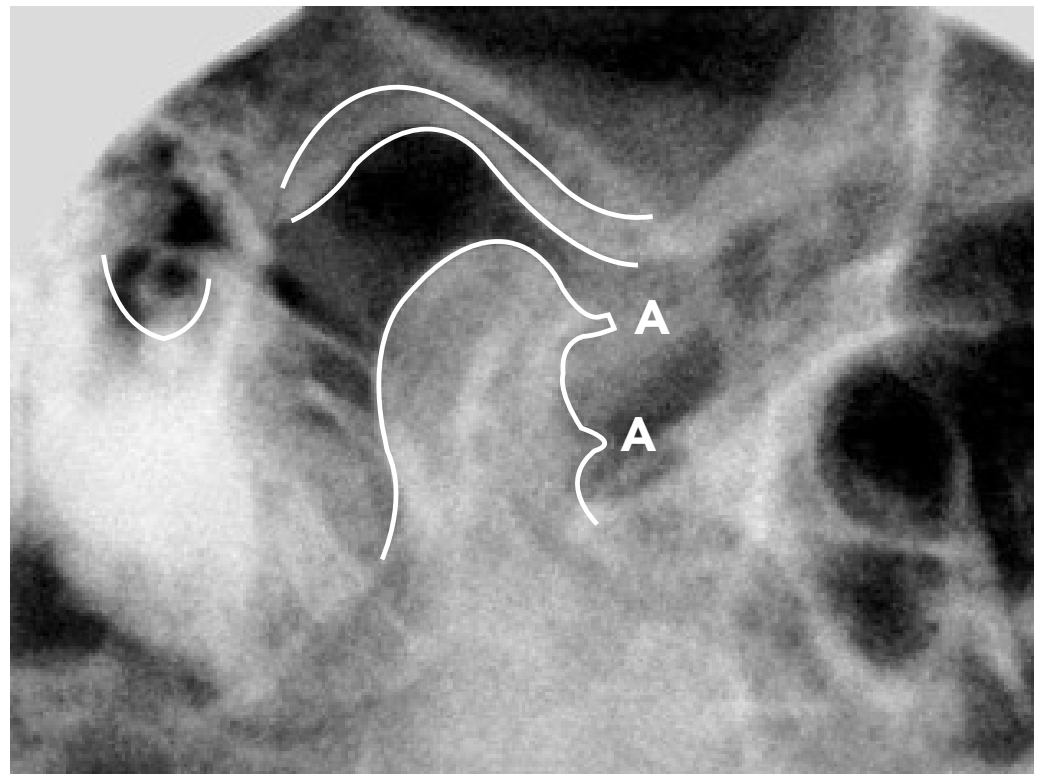

FIGURA 3 - A) Osteófito no côndilo mandibular.

\begin{tabular}{|c|c|c|c|c|c|c|}
\hline \multirow[b]{2}{*}{ Gênero } & \multicolumn{5}{|c|}{ Faixa Etária } & \multirow[b]{2}{*}{ Total } \\
\hline & $10|----| 19$ & $20|-----| 29$ & $30|---| 39$ & $40|----| 49$ & $\geq 50$ & \\
\hline Masculino & - & 2 & - & - & 1 & 3 \\
\hline Feminino & 1 & 6 & 1 & 3 & 3 & 14 \\
\hline Total & 1 & 8 & 1 & 3 & 4 & 17 \\
\hline
\end{tabular}

tados cruzados com o quadro clínico dos pacientes, em apenas um paciente do total (2 ATMs), não foi observado degenerações articulares.

\section{Metodologia estatística}

Através dos dados coletados fizemos uma análise cujos resultados são apresentados em formas de tabelas e/ou gráficos. Verificamos também, a correlação existente entre os achados radiográficos do tipo facetamento e eburnação, através do coeficiente de correlação não paramétrica de Sperman ${ }^{23}$.

\section{RESULTADOS}

A distribuição dos pacientes de acordo com gênero e faixa etária está referida na tabela 1 , onde observamos um predomínio de pacientes do gênero feminino (82,35\%). E destas, $43 \%$ possuem entre 20 e 29 anos de idade.

A distribuição das alterações degenerativas verificadas radiograficamente em cada articulação é observada na tabela 2. Foi observado um número maior do facetamento, principalmente no côndilo e, a eburnação foi mais significativa na eminência articular. A distribuição dos tipos de queixa principal em relação aos achados radiográficos por articulação é observada na tabela 3. Notamos um predomínio de dor pré-auricular e estalo associado ao facetamento. Os casos 


\begin{tabular}{|c|c|c|c|}
\hline \multicolumn{4}{|c|}{$\begin{array}{l}\text { Tabela } 2 \text { - Alterações degenerativas por } \\
\text { articulação observadas nos pacientes, radiograficamente. }\end{array}$} \\
\hline & \multicolumn{2}{|c|}{ Estrutura } & \multirow[b]{2}{*}{ Total } \\
\hline Alteração & Côndilo & Eminência Articular & \\
\hline Facetamento & 19 & 09 & 28 \\
\hline Eburnação & 03 & 07 & 10 \\
\hline Osteófito & 02 & - & 02 \\
\hline Total & 24 & 16 & 40 \\
\hline
\end{tabular}

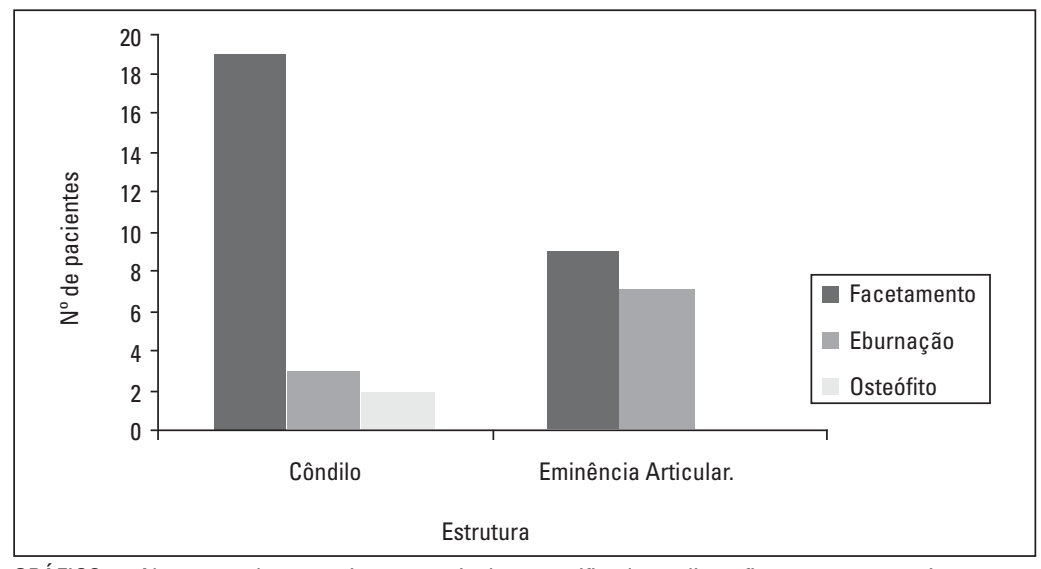

GRÁFICO 1 - Alterações degenerativas por articulação verificadas radiograficamente, nos pacientes.

\begin{tabular}{|c|c|c|c|}
\hline \multicolumn{5}{|c|}{ Tabela 3 - Tipo de queixa principal das } \\
\hline degenerações por articulação, encontradas radiograficamente. \\
\hline Queixa Principal & Achado Radiográfico \\
\hline Dor Pré-auricular & Facetamento & Eburnação & Osteófito \\
\hline Dor Muscular & 15 & 03 & 01 \\
\hline Má-oclusão & 06 & 02 & - \\
\hline Hábitos Parafuncionais & 09 & 04 & - \\
\hline Estalos & 07 & 02 & - \\
\hline Crepitação & 10 & 03 & - \\
\hline Limitação de Abertura Bucal & 09 & 04 & - \\
\hline Otalgia & 02 & - & 01 \\
\hline Zumbido & 03 & - & - \\
\hline Bruxismo & 02 & - & - \\
\hline Cefaléia & 02 & - & - \\
\hline Dor Cervical & 06 & 03 & - \\
\hline Luxação Recidivante & - & - & - \\
\hline
\end{tabular}

com osteófito foram associados à limitação da abertura bucal juntamente com dor pré-auricular. A distribuição dos pacientes de acordo com gênero e alterações degenerativas por articulação é verificada na tabela 4. A eburnação foi mais freqüente no gênero masculino enquanto o facetamento e o osteófito no gênero feminino.

Na tabela 2 e gráfico 1 constatamos um maior número de facetamento no côndilo, enquanto que na eminência articular tanto a eburnação quanto o facetamento apresentam número de casos não muito diferentes.

$\mathrm{Na}$ tabela 3 notamos um predomínio de dor pré-auricular e estalo associado ao facetamento. O osteófito está associado à limitação da abertura bucal juntamente com dor pré-auricular.

Podemos observar através da gráfico 2 que existe uma correlação de (70\%), entre os achados radiográficos do tipo, facetamento e eburnação, que são estatísticamente significantes ao nível de $5 \%$.

$\mathrm{Na}$ tabela 4 apresentamos a distribuição dos pacientes segundo o gênero e as alterações degenerativas por articulação, onde constatamos que o facetamento apresenta maior incidência (72\%) nas pacientes do gênero feminino, enquanto que a erbunação apresenta a mesma incidência tanto nos pacientes 


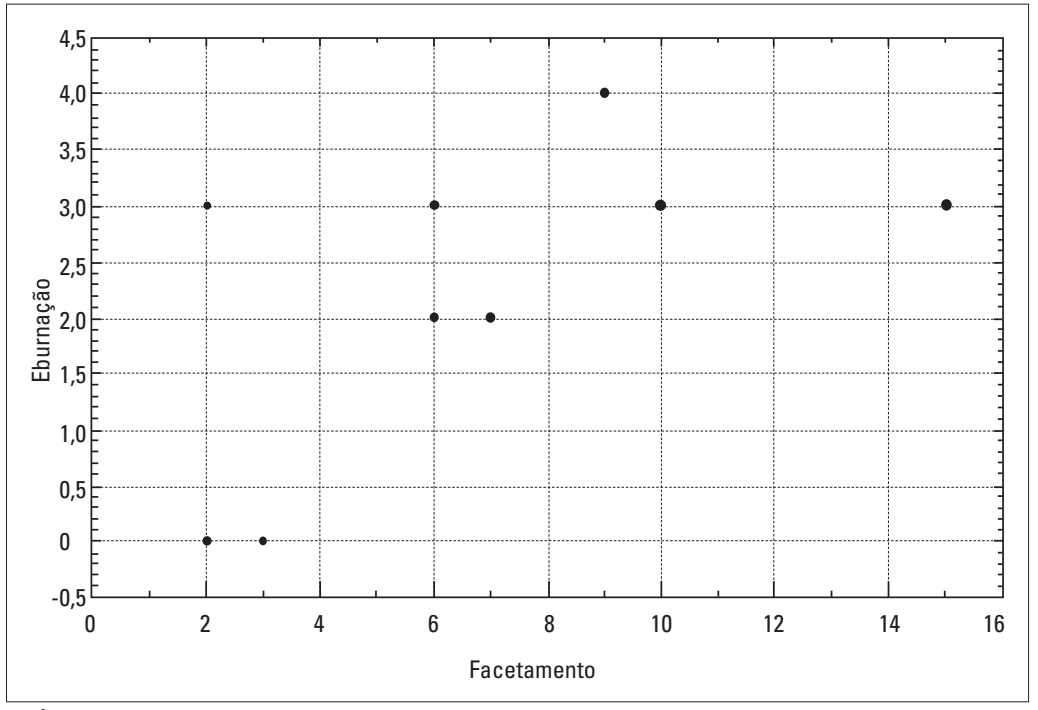

GRÁFICO 2 - Correlação entre os dados radiográficos facetamento e eburnação.

\begin{tabular}{|c|c|c|c|c|}
\hline Tabela 4 - Alterações degenerativas por articulação de acordo com o gênero. \\
\hline Gênero & Achado Radiográfico \\
\hline Masculino & 06 & 03 & 0 steófito & Total \\
\hline Feminino & 16 & 03 & - & 09 \\
\hline Total & 22 & 06 & 02 & 21 \\
\hline
\end{tabular}

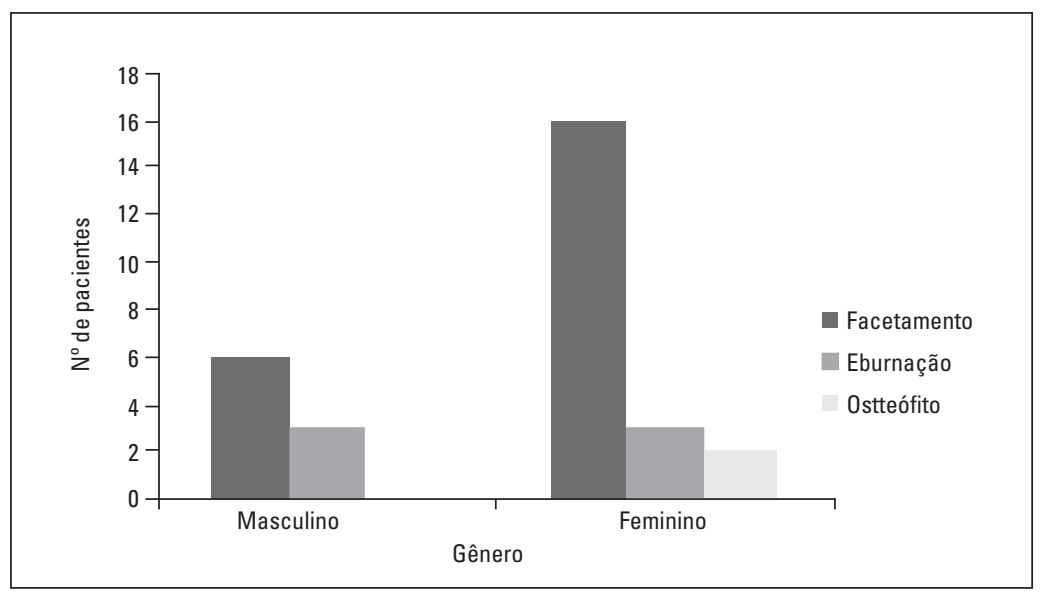

GRÁFICO 3 - Alterações degenerativas articulares segundo o gênero. do gênero masculino quanto feminino e o osteófito está presente somente em pacientes do gênero feminino. $O$ que é também demonstrado na gráfico 3. Sugere-se uma associação do achado radiográfico do tipo osteófito, com o gênero feminino, apesar de possuir apenas 02 casos e ser estatisticamente impossível sua análise.

\section{DISCUSSÃO}

Este estudo foi realizado através de incidência transcranial, pois trata-se da incidência radiográfica em que mais dados pode-se obter para avaliação da ATM, sendo menos onerosa e superada apenas pela tomografia linear ${ }^{2}$. É utilizada em muitos serviços públicos de Cirurgia Bucomaxilofacial em todo o país.

As mulheres são mais freqüentemente atingidas por essa síndrome que os homens. São atingidas na idade de 20 a 29 anos, (6 pacientes), sendo esses dados compatíveis com os estudos de Correia ${ }^{6}$; Lemos $^{13}$; Ra$\operatorname{mos}^{21}$, (Tab. 1). Alguns estudos envolvendo ratos relacionam um aumento dos sintomas de disfunção da ATM nas fêmeas devido ao estrógeno e à prolactina, que podem exacerbar a degradação da cartilagem e do osso além de estimular uma série de respostas imunes ${ }^{18}$. É importante a realização de estudos em seres humanos, sendo uma hipótese interessante 
de ser verificada.

Os achados radiográficos da ATM demonstraram neste estudo que os processos degenerativos (Tab. 2) estão de acordo com estudos similares realizados ${ }^{1,17}$. Os achados mais freqüentes foram o facetamento seguido de eburnação e o osteófito. No côndilo da mandíbula o facetamento foi mais freqüente enquanto que a eburnação foi mais efetiva na eminência articular sendo esses dados coerentes com os estudos de (PALÁCIOS-MORENO et al. ${ }^{19}$ ). Os achados deste trabalho são diferentes dos apresentados quando comparados aos artigos relacionados aos pacientes assintomáticos ${ }^{8,11}$ que referem técnicas radiográficas diferentes e maior número de degenerações do tipo osteófito.

$\mathrm{Na}$ análise das queixas principais relacionadas com os achados radiográficos dos pacientes, observamos significante presença da dor pré-auricular unilateral ou bilateral em nossos pacientes, assim como inúmeros trabalhos relacionam esse mesmo resultado ${ }^{7,9,13,19}$. Os resultados da dor pré-auricular estão diretamente relacionados com os achados degenerativos do tipo facetamento, como também relataram Ramos et al. ${ }^{21}$. Resultados diferentes foram verificados por Porto $^{20}$, que observou maior número do achado clínico o do tipo estalido em 18 ATMs estudadas através de ressonância magnética em pacientes usuários de dentaduras duplas.

O gênero feminino foi mais acometido pelas alterações estruturais da ATM. No gênero feminino foi comum a degeneração do tipo facetamento, tanto no côndilo quanto na eminência articular do osso temporal (Tab. 4).

No gênero masculino também foi freqüente o facetamento obtendo o mesmo número de eburnações das mulheres da amostra.

Os osteófitos compreenderam $6 \%$ do total da amostra apenas no côndilo da mandíbula. Ocorreram todos no gênero feminino, relacionados com queixa principal do tipo limitação da abertura bucal e dor pré-auricular. Foi encontrado um número de casos limitado para ser relacionado estatisticamente, mas permite uma análise mais detalhada deste tipo de alteração, para estudos futuros.

\section{CONCLUSÕES}

1) As alterações degenerativas ocorrem mais frequentemente no côndilo do que na eminência articular, em especial o facetamento.

2) A dor pré-auricular, estalos e crepitações foram as principais queixas dos pacientes.

3) Existe uma correlação de $70 \%$ entre o facetamento e a eburnação que são significantes estatisticamente ao nível de $5 \%$.

Enviado em: Outubro de 2003 Revisado e aceito: Janeiro de 2004 


\title{
Degenerative alterations in patients with craniomandibular desorders.
}

\begin{abstract}
The degenerative alterations of TMJ were study in 17 patients, 34 TMJs in the service of Hospital Jaraguá São Paulo-SP all of these patients with signs and symptoms of craniomandibular disfunction. It was used TMJ transcranial radiographs to verificate the joints degeneratives. The TMJs of each patient were examined by three differents professionals with help of radiographic exams. The clinical examination search to observe aspects as race, Sex, age the main complet of each patient, presence of pre-auricular pain, the click and limitation of mouth overture. The pre-auricular pain, the clicks were the main complete of the patients. The degenerative alterations were most frequent in woman on age between 20-29, in this decreansing order: flattenig, sclerosis, osteophyte. The alterations were more frequent in the condyle than in the articular eminence. It was observed estatisticaly a correlation of $70 \%$ between flattening and eburnation.Degenerative alterations were more frequent in the condyle than in the articular eminence. The flattening of the articular surfaces was relantionship with the articular pain.
\end{abstract}

Key words: Temporomandibular joint. Dysfunction. Radiology.

\section{REFERÊNCIAS}

1. AKERMAN, S.; KOPP, S.; ROHLIN, M. Macroscopic and microscopic appearance of radiologic findings in temporomandibular joints from elderly individuals. Int J Oral Maxillofac Surg, St. Louis, v. 17, p. 58-63, 1988.

2. ALFARO-SILVA, I.M.; FREITAS, A.; FENIO PEREIRA, M. Estudo anátomo-radiográfico da região têmporomandibular, com o emprego das técnicas radiográficas transfacial, transcranial e tomográfica. Rev Odontol Univ, São Paulo, v. 2, n. 4, p. 195200, out./dez. 1988.

3. BARROS, J. J. Os desarranjos da articulação temporomandibular e seu significado odontológico. Rev XXV Jan. [S.I.], v. 13, n. 42, p. 37-41, maio/jun. 1956.

4. BEAN, L. R.; OMNELL, K. A.; OEBERG, T. Comparison between radiologic observations and macroscopic tissue changes in temporomandibular joints. Dentomaxillofac Radiolol, Erlangen, v. 6, p. 90-106, 1977

5. CHILVALQUER, I. Imagenologia da ATM. In: BARROS, J. J. RODE, S. M. Tratamento das disfunções craniomandibulares: ATM. São Paulo: Ed. Santos, 1995. p. 129-151.

6. CORREIA, F. A. S. Análise da sintomatologia nas disfunções da articulação temporomandibular: contribuição ao estudo. São Paulo. 1991. 116 f. Tese (Livre-Docência)-Faculdade de Odontologia, Universidade de São Paulo, São Paulo, 1991.

7. COSTEN, J. B. A syndrome of car and sinus symptons dependent upon disturbed function of the temporomandibular joint Ann Otol, St. Louis, v. 43, no. 1, p.1-15, Mar. 1934
8. ERICSON, S.; LUNDENBERG, M. Structural changes in the finger, wrist and temporomandibular joints. A comparative radiologic study. Acta Odontol Scand, Stockholm, v. 26, no. 2 , p. 111-125, May 1968.

9. FARRAR, W. B. Differentiation of temporomandibular joint dysfunction to simplify treatment. J Prosth Dent, St. Louis, v. 28 , no. 6, p. 629-636, Dec. 1972.

10. HELKIMO, M. Studies on function and dysfunction of the mastigatory system. I An epidemiological investigation of synptoms of dysfunction in Lapps in The North of Finland. Proc Finn Dent Soc, Helsinki, v. 47, no. 2, p. 37-49, Apr. 1974.

11. ISBERG, A.; ISACSSON, G. Tissue reactions associated with internal derangement of the temporomandibular joint. A radiographic, criomorphologic, and histologic study. Acta Odontol Scand, Stockholm, v. 44, no. 3, p. 159-163, jan. 1986.

12. LEANDRO, L. F. L.; NUNES, L. J. ATM: diagnóstico e tratamento. São Paulo: Pancast, 2000.

13. LEMOS, J. B. D. Avaliação da excursão do côndilo da mandíbula e sua relação com a sintomatologia, em pacientes portadores da síndrome dor-disfunção da articulação tem poromandibular. 1988. 98 f. Dissertação (Mestrado)- Faculdade de Odontologia, Universidade de São Paulo, São Paulo, 1988

14. MAGNUSSON, T. ; KARLSSON, C. Clinical impact of radiological examinations of patients with suspected temporomandibular disorders. Swed Dent J, Stockholm, v. 26, no. 2, p. 67-74 2002. 
15. MCQUEEN, W. W. Radiography of temporomandibular articulation. Minn Dist Dent J, [S. I.], v. 21, no. 9, p. 28-30, Sept. 1937.

16. MORAES, L.C. de; DUARTE, M. S. R.; MEDICI FILHO, E.;

MORAES, M. E. L. de. Imagens da ATM: técnicas de exame. J Bras Ortodon Ortop Facial, Curitiba, v. 6, n. 36, p. $502-507$, nov./dez. 2001.

17. MUIR, C. B.; GOSS, A. N. The radiologic morphology of painfull temporomandibular joints. Oral Surg Oral Med Oral Pathol, St. Louis, v. 70, no. 3, p. 355- 359, Sept. 1990.

18. OKUDA, T. Effect of estrogen replacement on temporomandibular joint remodeling in ovariectomized rats. Department of Oral and Maxillofacial Surgery, Gifu University School of Medicine, Japan. J Oral Maxillofac Surg, St. Louis, v. 58, no. 2, p. 189-196, 196-197, Feb. 2000.

19. PALÁCIOS-MORENO, A. M.: CHILVALQUER, I.: LUZ, J. G. C. Achados radiográficos, sinais e sintomas nas disfunções da articulação temporomandibular. Rev Odontol Univ São Paulo, São Paulo, v.11, n. 4, p. 273-278, out./dez. 1997.
20. PORTO , C. V. Avaliação da posição do disco articular em pacientes usuários de dentaduras duplas e portadores de sons articulares, por meio de imagem de ressonância magnética da ATM. 2002116 p. Tese (Doutorado)- Faculdade de Odontologia de Bauru, Universidade de São Paulo, Bauru, 2002.

21. RAMOS, H. A. D. Sinais e sintomas das disfunções dolorosas da articulação temporomandibular. Cad Documento, São Bernardo do Campo, v. 2, n. 2, p. 252-255, jul./ago. 1992.

22. RICKETTS, R. W. Variations of the temporomandibular joint as revealed by cephalometric laminagraphy. Am J Orthod, St. Louis, v. 36, no. 120, p. 877-899, 1950.

23. SIEGEL, S. Estatística não paramétrica. São Paulo: McGrawHill, 1975.

Endereço para correspondência

João Baptista Ilha Filho.

R. Moisés Marcondes 115,

CEP: 87010-390 - Maringá-PR, Brasil.

joilha@globo.com 